$\underset{\text { clinical }}{\text { nephron }}$

Practice
Nephron 2018;140:90-93

DOI: 10.1159/000491557
Received: April 30, 2018

Accepted after revision: June 25, 2018

Published online: July 11, 2018

\title{
Ventilator-Induced Kidney Injury: Are Novel Biomarkers the Key to Prevention?
}

\author{
Mark L. Hepokoski ${ }^{a, c}$ Atul Malhotra ${ }^{a}$ Prabhleen Singh ${ }^{b, c}$ \\ Laura E. Crotty Alexander ${ }^{a, c}$ \\ ${ }^{a}$ Division of Pulmonary and Critical Care Medicine, University of California San Diego, La Jolla, CA, USA; \\ ${ }^{b}$ Division of Nephrology and Hypertension, University of California San Diego, San Diego, CA, USA; \\ 'VA San Diego Health Systems, San Diego, CA, USA
}

\section{Keywords}

Ventilator-induced kidney injury · Urine biomarkers

\section{Abstract}

Mechanical ventilation is associated with significant increases in the risk of acute kidney injury (AKI). The rate of AKI due to mechanical ventilation and the associated mortality remain unacceptably high. Preventative and therapeutic strategies are clearly lacking. Ventilator-induced kidney injury is believed to occur due to changes in hemodynamics that impair renal perfusion, neurohumoral-mediated alterations in intra-renal blood flow, and systemic inflammatory mediators generated by ventilator-induced lung injury. The risk of injury to the kidney by these mechanisms may be modified by open lung protective ventilation with low tidal volumes and high positive end expiratory pressure. However, these strategies may also increase the risk of injury in some settings, and clinicians have limited means to identify the optimal ventilator strategy for each specific patient. Novel urinary biomarkers have demonstrated the ability to predict AKI prior to classic clinical signs such as decreased urine out- put and increased creatinine. These biomarkers may serve as an early indication to intensivists of an injurious ventilator strategy and failure of traditional management.

(c) 2018 S. Karger AG, Basel

\section{Introduction}

Mechanical ventilation is often required as a life-saving intervention in critically ill patients. However, mechanical ventilation also increases the risk of acute kidney injury (AKI) threefold [1]. The consequences of AKI in mechanically ventilated patients are important, as the independent mortality of AKI in the intensive care unit (ICU) is $30-50 \%$ [2] and patients with combined respiratory fail-

Contribution from the AKI and CRRT 2018 Symposium at the 23rd International Conference on Advances in Critical Care Nephrology, Manchester Grand Hyatt, San Diego, CA, USA, February 26-March 1,2018 . This symposium was supported in part by the NIDDK funded University of Alabama at Birmingham-University of California San Diego O'Brien Center for Acute Kidney Injury Research (P30DK079337).

\section{KARGER}

(C) 2018 S. Karger AG, Basel

E-Mail karger@karger.com

www.karger.com/nef
Dr. Mark L. Hepokoski

Division of Pulmonary and Critical Care Medicine

University of California San Diego

9300 Campus Point Drive, \#7381, La Jolla, CA 92037-7381 (USA)

E-Mail mhepokoski@ucsd.edu 
ure and AKI have a mortality of $60-80 \%$ [3]. Extensive research into the systemic effects of mechanical ventilation has provided important insights into the potential mechanisms of ventilator-induced kidney injury (VIKI). Unfortunately, the rate of AKI in mechanically ventilated patients remains unacceptably high, and preventative and therapeutic strategies are very limited. In this review, we describe our current knowledge regarding mechanisms of VIKI, and discuss the potential role of novel urinary biomarkers as a means to guide intensivists in choosing a renal-protective mechanical ventilation strategy.

\section{Mechanisms and Current Approach to Mechanical Ventilation-Induced Kidney Injury}

The adverse renal effects of mechanical ventilation have been known for over 70 years. In 1947, Drury et al. [4] were the first to our knowledge to investigate this notion by measuring urea clearance in human subjects exposed to varying levels of continuous positive airway pressure. They observed a clear, inverse relationship between the level of continuous positive airway pressure applied and kidney function, and the authors concluded that mechanical ventilation induced a "circulatory stress" that could be identified by reduced renal function. Subsequent studies in canines helped elucidate the mechanisms involved by showing that positive pressure ventilation causes a reduction in cardiac output by impeding venous return [5]. It was discovered later that neurohumoral mediators released during mechanical ventilation also adversely alter renal blood flow from cortex to medulla, which leads to sodium reabsorption and a reduction in the glomerular filtration rate. Sodium reabsorption in the kidney requires high oxygen utilization [6]; therefore, mechanical ventilation may decrease oxygen delivery through systemic hemodynamic effects while subsequently increasing oxygen utilization via neurohumoral mediators.

More recently, ventilator-induced lung injury (VILI) has been proposed as another mechanism of AKI via inflammatory crosstalk from the lung to kidney [7]. Although mechanical ventilation has a major role in life support, evidence suggests that certain ventilator settings may induce lung injury in some cases or may worsen lung injury once established [8]. Positive pressure ventilation using high tidal volumes with associated high transpulmonary pressures can stretch the lung and lead to damage of the alveolar capillary membrane (i.e., volutrauma and barotrauma). Additionally, some lung units, particularly those with high surface tension, may be at risk of lung in- jury due to repetitive alveolar collapse and reopening (i.e., atelectrauma) [8]. VILI leads to damaged pulmonary epithelial and endothelial cells that activate innate immune cascades resulting in the release of proinflammatory cytokines, such as IL- 6 and TNF- $\alpha$ [8]. These cytokines may then enter the systemic circulation and propagate inflammatory cascades in the kidney in a process called biotrauma [8]. Imai et al. [9] demonstrated that biotrauma is a likely mechanism of VIKI by showing that serum from rabbits subjected to injurious, high tidal volume ventilation led to renal apoptosis in vitro.

Open lung protective ventilation refers to a ventilation strategy aimed at preventing VILI and systemic biotrauma with low tidal volumes to prevent alveolar overdistention and high positive end expiratory pressure (PEEP) to prevent atelectrauma. In 2000, the Acute Respiratory Distress Syndrome Network investigators published the landmark ARMA study, which showed that this open lung protective ventilation strategy improved renal outcomes as measured by renal failure-free days and reduced mortality in patients with Acute Respiratory Distress Syndrome [10]. Notably, a recent systematic review and meta-analysis revealed that mechanical ventilation remains an independent risk factor for AKI despite the ARMA ventilation protocol [1], and no other therapies currently exist. One of the barriers to the development of novel therapies is the fact that it remains unclear from epidemiologic studies if modern mechanical ventilation is in fact a "cause" of AKI rather than an association. For example, it is possible that the higher rates of AKI in mechanically ventilated patients are simply due to common comorbid disease processes or therapies, such as, sedation and antibiotics or simply a marker of severity of illness. Causality is strongly suggested by the fact that even modest amounts of positive pressure applied to previously healthy canines or humans causes a drop in urine output $[4,5]$, but the exact mechanisms involved have not been completely elucidated. Further research in this area is clearly needed to prove causality and to identify potentially modifiable therapeutic targets.

\section{Urine Biomarkers in the Early Detection of VIKI and "Patient-Specific" Mechanical Ventilation}

Currently, the ARMA protocol is applied to almost all patients in the ICU as a "one size fits all" approach to mechanical ventilation. However, recent data have suggested that the tidal volumes used in this protocol $(6-8 \mathrm{~mL} / \mathrm{kg}$ idealbody weight) may still lead to VILI in some patients, while 
others may actually do better with slightly higher tidal volumes due to the higher levels of sedation required to maintain low tidal volumes [11]. The "best PEEP" to prevent atelectrauma while maintaining adequate renal perfusion is also unclear. Therefore, many intensivists believe that a more patient-specific approach may be better, and there is extensive work focusing on novel measurements that could guide clinicians in choosing the most appropriate ventilator settings for each specific patient [11]. For example, if clinicians were aware that the current ventilator settings they were utilizing in a particular patient were adversely affecting the kidney, they would almost certainly consider a change. Recently, several novel urinary biomarkers have demonstrated the ability to predict AKI prior to clinical detection. The utility of these biomarkers in preventing VIKI remains to be established, but the information provided could be extremely valuable to intensivists as real-time output following the initiation of mechanical ventilation.

The urinary concentration of the tissue inhibitor of metalloproteinase 2 multiplied by the concentration of insulin-like growth factor binding protein 7 has been shown to predict accurately the development of moderate-to-severe AKI in ICU patients with respiratory or cardiovascular impairment $[12,13]$. This combination of biomarkers is becoming widely utilized in ICUs around the world, and their ability to lead to AKI prevention looks promising. Other urinary biomarkers that show promise are those based on renal mitochondrial injury discovered by Whitaker et al. [14, 15]. These investigators used animal models to show that the mitochondrial byproducts, mitochondrial DNA [15] and ATP Synthase Subunit- $\beta$ [14], are released into the urine following just 10 minutes of renal ischemia and reperfusion. They showed later that urinary levels of these mitochondrial fragments predict the development of AKI in human subjects after cardiothoracic surgery. Mitochondrial-based biomarkers may have particular utility in predicting VIKI, as it makes mechanistic sense that VIKI would promote mitochondrial injury. As previously stated, mechanical ventilation causes changes in renal blood flow that may simultaneously decrease oxygen delivery and increase oxygen consumption. This imbalance in oxygen delivery utilization would be expected to cause profound renal mitochondrial injury that is likely to occur prior to reduced urine output and creatinine elevation.

Enthusiasm for these biomarkers is tempered by the fact that interventional trials demonstrating improved outcomes are lacking. The prevention of VIKI offers a unique opportunity for an interventional trial, as it is not often that clinicians know exactly when a renal stress will occur (e.g., the initiation of positive pressure ventilation). Urinary biomarker levels could be evaluated prior to and after the initiation of mechanical ventilation, and physicians could change or maintain ventilator settings or consider additional hemodynamic support based on the results. These biomarkers could also be used in combination with lung injury scores and lung mechanics assessments to identify those patients who are not responding to traditional open lung protective ventilation, and may require early neuromuscular blockade, prone positioning, or extra corporeal membrane oxygenation.

\section{Conclusion}

AKI occurs commonly in mechanically ventilated patients and the associated mortality rate is exceptionally high. Despite the important advances in our knowledge about lung-kidney interactions, a number of questions remain regarding the exact mechanisms involved in VIKI. Given the major clinical impact, the myriad of open questions for clinicians and scientists, and the unique opportunity for preventative therapies, we advocate for further focus on this topic. Novel therapeutic strategies would be a welcome addition to our armamentarium and will likely require a multidisciplinary approach with the involvement of nephrologists, pulmonologists, scientists, and intensivists.

\section{Acknowledgments}

Dr. Mark L. Hepokoski is supported by NIH T32 DK104717. Dr. Atul Malhotra is PI on NIH RO1 HL085188, K24 HL132105, and T32 HL134632 and co-investigator on R21 HL121794, RO1 HL119201, and RO1 HL081823. As an Officer of the American Thoracic Society, Dr. Atul Malhotra has relinquished all outside personal income since 2012. ResMed, Inc. provided a philanthropic donation to the UC San Diego in support of a sleep center. Dr. Prabhleen Singh is PI on Veterans Affairs (VA) Merit Award BX002175 and NIH R03 DK101841. Dr. Crotty Alexander is PI on American Heart Association Beginning Grant-in-Aid 16BGIA27790079, UAB-UCSD O'Brien Center Daniel O'Connor Scholar Award NIH P30-DK079337, VA BLR\&D Career Development Award 1IK2BX001313, and an ATS Foundation Award. The content is solely the responsibility of the authors and does not necessarily represent the official views of the funding sources.

\section{Disclosure Statement}

The authors declare that there are no competing financial interests.
92

Nephron 2018;140:90-93

DOI: $10.1159 / 000491557$
Hepokoski/Malhotra/Singh/

Crotty Alexander 


\section{References}

1 van den Akker JP, Egal M, Groeneveld AB: Invasive mechanical ventilation as a risk factor for acute kidney injury in the critically ill: a systematic review and meta-analysis. Crit Care 2013;17:R98.

2 Chawla LS, Amdur RL, Shaw AD, Faselis C, Palant CE, Kimmel PL: Association between $\mathrm{AKI}$ and long-term renal and cardiovascular outcomes in United States veterans. Clin J Am Soc Nephrol 2014;9:448-456.

3 Chertow GM, Christiansen CL, Cleary PD, Munro C, Lazarus JM: Prognostic stratification in critically ill patients with acute renal failure requiring dialysis. Arch Intern Med 1995; 155:1505-1511.

4 Drury DR, Henry JP, Goodman J: The effects of continuous pressure breathing on kidney function. J Clin Invest 1947;26:945-951.

5 Priebe HJ, Heimann JC, Hedley-Whyte J: Mechanisms of renal dysfunction during positive end-expiratory pressure ventilation. J Appl Physiol Respir Environ Exerc Physiol 1981;50:643-649.

6 Bullen A, Liu ZZ, Hepokoski M, Li Y, Singh P: Renal oxygenation and hemodynamics in kidney injury. Nephron 2017;137:260263.

7 Hepokoski M, Englert JA, Baron RM, Crotty-Alexander LE, Fuster MM, Beitler JR, Malhotra A, Singh P: Ventilator-induced lung injury increases expression of endothelial inflammatory mediators in the kidney.
Am J Physiol Renal Physiol 2017;312:F654F660.

8 Slutsky AS, Ranieri VM: Ventilator-induced lung injury. N Engl J Med 2014;370:980.

9 Imai Y, Parodo J, Kajikawa O, de Perrot M, Fischer S, Edwards V, Cutz E, Liu M, Keshavjee S, Martin TR, Marshall JC, Ranieri VM, Slutsky AS: Injurious mechanical ventilation and end-organ epithelial cell apoptosis and organ dysfunction in an experimental model of acute respiratory distress syndrome. JAMA 2003;289:2104-2112.

10 Acute Respiratory Distress Syndrome Network, Brower RG, Matthay MA, Morris A, Schoenfeld D, Thompson BT, Wheeler A: Ventilation with lower tidal volumes as compared with traditional tidal volumes for acute lung injury and the acute respiratory distress syndrome. N Engl J Med 2000;342:1301-1308.

11 Hepokoski M, Owens RL, Malhotra A, Beitler JR: Mechanical ventilation in acute respiratory distress syndrome at ATS 2016: the search for a patient-specific strategy. J Thorac Dis 2016;8(suppl 7):S550-S552.

12 Kashani K, Al-Khafaji A, Ardiles T, Artigas A, Bagshaw SM, Bell M, Bihorac A, Birkhahn R, Cely CM, Chawla LS, Davison DL, Feldkamp T, Forni LG, Gong MN, Gunnerson KJ, Haase M, Hackett J, Honore PM, Hoste EA, Joannes-Boyau O, Joannidis M, Kim P, Koyner JL, Laskowitz DT, Lissauer ME, Marx G, McCullough PA, Mullaney S, Oster- mann M, Rimmele T, Shapiro NI, Shaw AD, Shi J, Sprague AM, Vincent JL, Vinsonneau C, Wagner L, Walker MG, Wilkerson RG, Zacharowski K, Kellum JA: Discovery and validation of cell cycle arrest biomarkers in human acute kidney injury. Crit Care 2013; 17:R25.

13 Bihorac A, Chawla LS, Shaw AD, Al-Khafaji A, Davison DL, Demuth GE, Fitzgerald R, Gong MN, Graham DD, Gunnerson K, Heung M, Jortani S, Kleerup E, Koyner JL, Krell K, Letourneau J, Lissauer M, Miner J, Nguyen HB, Ortega LM, Self WH, Sellman R, Shi J, Straseski J, Szalados JE, Wilber ST, Walker MG, Wilson J, Wunderink R, Zimmerman J, Kellum JA: Validation of cell-cycle arrest biomarkers for acute kidney injury using clinical adjudication. Am J Respir Crit Care Med 2014;189:932-939.

14 Whitaker RM, Korrapati MC, Stallons LJ, Jesinkey SR, Arthur JM, Beeson CC, Zhong Z, Schnellmann RG: Urinary ATP synthase subunit $\beta$ is a novel biomarker of renal mitochondrial dysfunction in acute kidney injury. Toxicol Sci 2015;145:108-117.

15 Whitaker RM, Stallons LJ, Kneff JE, Alge JL, Harmon JL, Rahn JJ, Arthur JM, Beeson CC, Chan SL, Schnellmann RG: Urinary mitochondrial DNA is a biomarker of mitochondrial disruption and renal dysfunction in acute kidney injury. Kidney Int 2015;88: 1336-1344 